\title{
Plasticity and Decomposition of Whiskers on Electric-Induced Deformation
}

\author{
A. P. Rodzevich ${ }^{1, \text { a) }}$, L. V. Kuzmina ${ }^{2, \text { b) }}$, E. G. Gazenaur ${ }^{2, \text { b) }}$, \\ and V. I. Krasheninin ${ }^{2, b)}$ \\ ${ }^{1}$ Yurga Institute of Technology, Tomsk Polytechnic University, Yurga, 652055, Russia \\ ${ }^{2}$ Kemerovo State University, Kemerovo, 650043, Russia \\ a) Corresponding author: nimez@tpu.ru \\ ${ }^{b)}$ specproc@kemsu.ru
}

\begin{abstract}
The purpose of the work is to study the influence of weak electric fields $(1-10 \mathrm{~V} / \mathrm{cm})$ on the micro and macroplasticity of whisker crystals of silver azide. The paper considers the processes occurring in the crystals of silver azide on the indentation in noncontact electric field. One of the processes is the indenter-induced movement of unfixed dislocations, which is the evidence of crystals microplasticity, followed by the accumulation of dislocations at the impediments of different nature. Further, this causes the change in linear dimensions of the crystals and the return to original dimensions, which is the evidence of macroplasticity. After deformation, a greater number of dislocations are formed, which stops the formation of the reaction areas where outgassing is observed. A method for controlling the whiskers stability and reactivity using the microindentation in electric field is proposed.
\end{abstract}

Keywords: plasticity, deformation, solid-phase decomposition, electric field, edge dislocation

\section{INTRODUCTION}

The problem arising on studying the effect of electromagnetic field on physicochemical properties of heavy metal azides (HMA) becomes significant when considering the methods for controlling the environmental stability of such systems during long-term storage.

The necessity and relevance of studying the plasticity of silver azide whiskers as HMA representative exposed to energy deposition is dictated by both scientific importance of this problem and practical demands of its solution.

Existing papers that associate the decomposition of azides with their plastic and strength properties state that such properties vary under different types of exposure (electric and magnetic fields, radiation) [1-3]. In all the cases, both microplasticity (the change of dislocation structure over time), and macroplasticity (the change in linear dimensions) are observed. The analysis of previously obtained experimental data shows that the outcropping of dislocations on the crystal surface coincide with the locations of intensive outgassing, while the decomposition reaction is triggered in the reaction areas formed by fixed dislocations [3]. The decomposition reaction is preceded by a phenomenon of crystal resizing [2].

From the microscopic point of view, the plastic deformation of crystals is a results of moving linear defects or dislocations. Since the dislocations in silver azide have charges [3, 4], the study of the electric field impact on the plastic deformation process is reasonable. Structural defects determining the mechanical properties of materials have electric charges and immediately respond to electric field exposure or electric current flow. This is particularly important for small objects. They can not only gradually change their state but even collapse.

Investigations were carried out using the whiskers of silver azide as a traditional model compound in solid state chemistry. The relevance of studying the whiskers increases when it comes to the information about properties of explosive substances for which the use of conventional mechanical tests on macro-samples is hindered. 


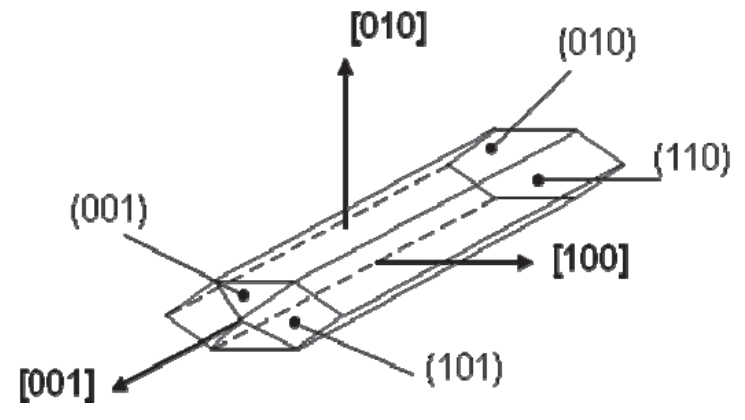

FIGURE 1. Indices of crystal faces and directions in silver azide whisker

The purpose of the paper is to study the influence of weak electric fields $(1-10 \mathrm{~V} / \mathrm{cm})$ on the micro-and macroplasticity of whisker crystals of silver azide.

\section{EXPERIMENTAL}

Silver azide whiskers were used as the objects of study; the crystals were grown by the method proposed by F.I. Ivanov [5] and that involves the slow evaporation of solvent of 5\% aqueous ammonia solution, the average dimensions of the crystal being equal to $10 \times 0.1 \times 0.03 \mathrm{~mm}$ (Fig. 1).

To change the density of dislocations the indentation technique was used. We have used a tungsten indenter (radius is $10 \mathrm{~mm}$, a localized pressure of $\left.\left(5 \cdot 10^{5}\right) \tilde{-}\left(3 \cdot 10^{7}\right) \mathrm{N} / \mathrm{m}^{2}\right)$ for analyzing the sample by a microscope with 120x magnification. To observe the outgassing at local indentation in noncontact electric field the sample was placed in a Plexiglas box which served as a condenser lined with copper plates (the height of electrodes was $0.5 \mathrm{~cm}$, crystal thickness was $0.003 \mathrm{~cm}$, which allowed creating a uniform electric field). This configuration was placed on the microscope stage (Fig. 2). The indenter was located at one end of the crystal coated with a layer of paraffin oil. The density of etch pits as well as the alteration of the sample size and the escape of gaseous products (gas bubbles of nitrogen) were analyzed using the microscope. The gas volume and escape rate into the oil were also determined.

As a UV-radiation source a DRSh-100 mercury lamp was used. The light intensity was $1.924 \cdot 10^{15}$ quanta $/\left(\mathrm{cm}^{2} \cdot \mathrm{s}\right)$. All experiments were performed at room temperature. For each point of the experimental curves at least 10 measurements were made.

\section{RESULTS AND DISCUSSION}

The study included the analysis of the changes in the density of edge dislocations in silver azide crystals under mechanical load (microindentation) for every sample. After increasing the indentation time we detected the change in the dislocation density. This accumulation process goes faster under high mechanical stresses, and the sample fails faster. The indenter pressure at which the sample stays intact longer is $5 \cdot 10^{5} \mathrm{~N} / \mathrm{m}^{2}$ (Fig. 3).

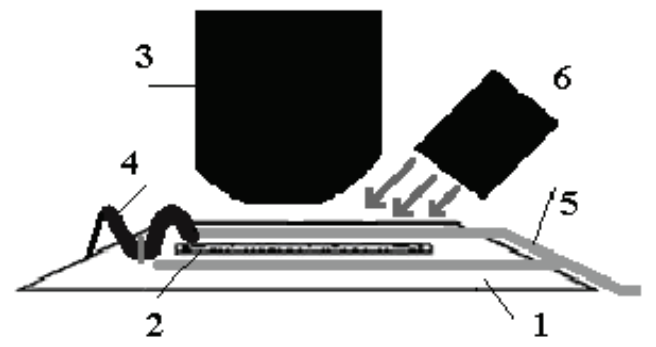

FIGURE 2. Scheme of experiment on external gassing of silver azide crystals: glass substrate (1), crystal (2), microscope (3), tungsten indenter with the given spring stiffness (4), copper electric contacts (5), UV light source (6) 


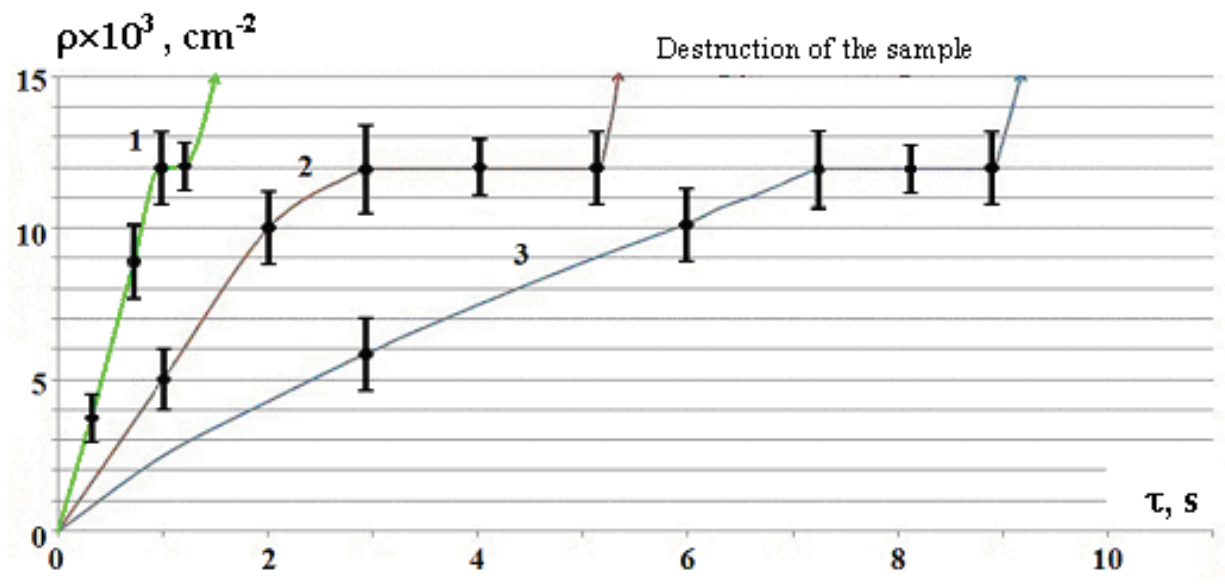

FIGURE 3. Dependence of the density of dislocations in silver azide crystals on strain exposure time: $3 \cdot 10^{7}(1), 3 \cdot 10^{6}(2), 5 \cdot 10^{5} \mathrm{~N} / \mathrm{m}^{2}(3)$

Thus, we have experimentally determined that exceeding the mechanical stress of about $10^{7} \mathrm{~N} / \mathrm{M}^{2}$ leads to the failure of silver azide crystals.

We were the first to observe in the experiment the decomposition outgassing on microindentation of silver azide whiskers. Quantitatively, the decomposition outgassing process is plotted as the dependence of escaped gas volume on the time of the indenter exposure (Fig. 4).

It has been established that the greater mechanical stress is applied, the less time is required for the emission of gaseous decomposition products. This fact should be taken into account especially when the impacts of several types are applied, one of them being a mechanical impact that increases the density of dislocations. One should note the relationship between the dislocation density and gas emission intensity, namely, the dislocation density being equal to the reaction area density. Correspondingly, the more the reaction area is, the more is the intensity of the crystal decomposition.

We have established the impact of a contactless electric field on the dislocation density and dimensional changes in microindentation of silver azide crystals. The contactless electric field stimulates the movement of edge dislocations and their resizing under the microindentation of silver azide crystals.

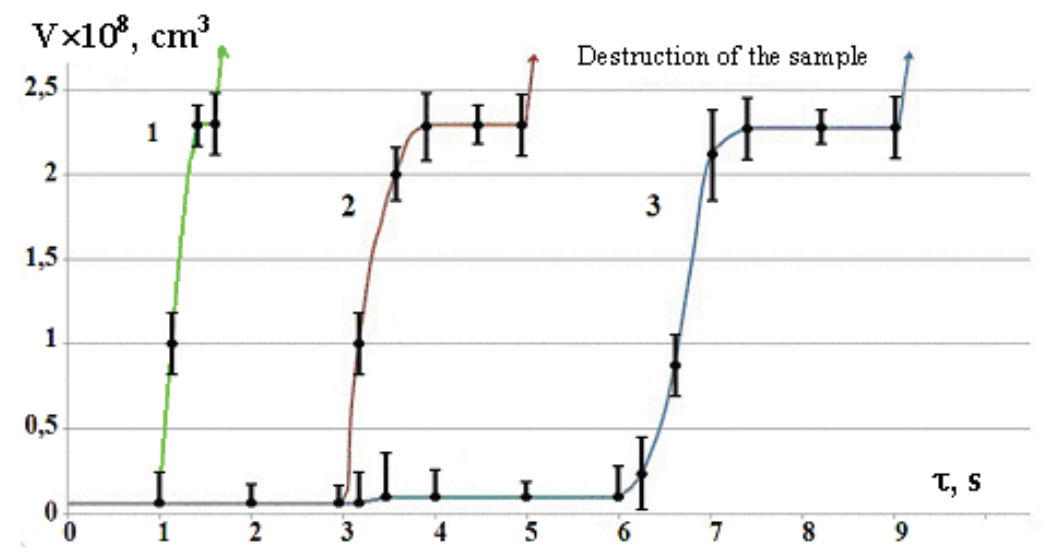

FIGURE 4. Dependence of gas released volume in silver azide crystals on time of mechanical action (indentation) at different indenter stress: $3 \cdot 10^{7}(1), 3 \cdot 10^{6}(2), 5 \cdot 10^{5} \mathrm{~N} / \mathrm{m}^{2}(3)$ 


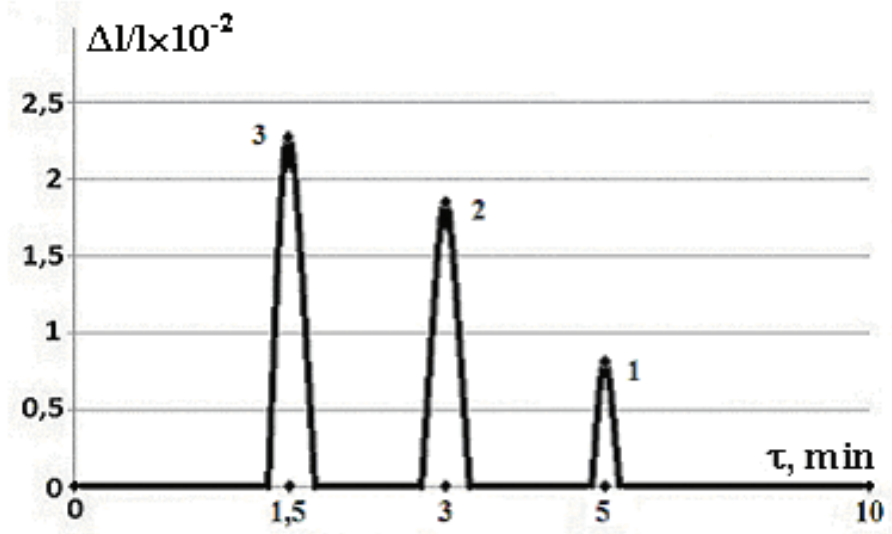

FIGURE 5. Dependence of relative change in silver azide crystals size along [100] axis on the indentation time in noncontact electric field: $1-5 \mathrm{~V} / \mathrm{cm}^{2}, 2-7 \mathrm{~V} / \mathrm{cm}^{2}, 3-10 \mathrm{~V} / \mathrm{cm}^{2}$

Let us consider the processes occurring in silver azide crystals under indentation in noncontact electric field. One of them is the movement of unfixed dislocations, imposed by the indenter, which evidences the microplasticity of crystals. Initially, the accumulation of dislocations at the impediments of different nature (usually impurity atoms, vacancies) occurs then followed by a gradual retardation.

Under the conditions of the experiment the dislocations imposed by the indenter were constantly moving under the electric field exposure (this was proved by the absence of etch pits and the emission of gaseous products). Therefore, in our case, we can assume that the field force affects the negatively charged edge dislocations (dislocation line charge is $10^{-16} \mathrm{C}$ [4]). Moreover, there is an evidence of macroplasticity stated by the change in linear dimensions of the crystals: after the maximum change in linear dimensions $\Delta l / l=(2.5 \pm 0.5) \cdot 10^{-2}$ a sharp decrease to the original dimensions was observed (Fig. 5).

Without external exposure the crystal relaxes approximately in one day. The noticeable change in the size is observed along the direction of electric field vector. Deformation, i.e. the change in crystal size, is observed after all entered dislocation have come to the surface under the electric field exposure. The deformation is followed by the formation of more dislocations that will interact with each other impeding the movement of each other, i.e. dislocations will stop and form reaction areas from which the gas emission is observed.

Based on the obtained results, a Control Method for Whiskers Stability and Reactivity using a microindentation in the electric field can be proposed.

\section{REFERENCES}

1. V. Yu. Zakharov, V. I. Krasheninin, L. V. Kuz'mina, and Yu. A. Zakharov, Solid State Ionics 101-103, 161 (1997).

2. V. E. Khramchenko, L. V. Kuz'mina, and V. I. Krasheninin, Proc. Fourth Int. Conf. "Single Crystal Growth and Heat and Mass Transfer”, Obninsk, 2001 (SSC RF IPPE, Obninsk, 2001), Vol. 3, p. 559.

3. V. I. Krasheninin, L. V. Kuzmina, and V. E. Ivaschenco, Mater. Res. Innovat. 5, 219 (2002).

4. L. V. Kuzmina, E. V. Sugatov, E. G. Gazenaur, and V. I. Krasheninin, Modern Problems of Science and Education 6 (2013), see http://www.science-education.ru/113-11757.

5. V. I. Krasheninin, L. V. Kuz'mina, V. Yu. Zakharov, and A. Yu. Stalinin, Chem. Phys. Reports 4, 529 (1995). 